\title{
Forecasting time and place of earthquakes using a Semi-Markov model (with case study in Tehran province)
}

\author{
Ramin Sadeghian
}

\begin{abstract}
The paper examines the application of semi-Markov models to the phenomenon of earthquakes in Tehran province. Generally, earthquakes are not independent of each other, and time and place of earthquakes are related to previous earthquakes; moreover, the time between earthquakes affects the pattern of their occurrence; thus, this occurrence can be likened to semi-Markov models. In our work, we divided the province of Tehran into six regions and grouped the earthquakes regarding their magnitude into three classes. Using a semi-Markov model, it proceeds to predict the likelihood of the time and place of occurrence of earthquakes in the province.
\end{abstract}

Keywords: Semi-Markov model, Earthquake occurrence, Forecasting, Transition matrix

\section{Background}

Forecasting the time and place of earthquakes is considered to be very important in science (Sadeghian 2007). Many researchers in the fields of mathematics, physics, and geology have tried to forecast the time and place of earthquake occurrence. Most of these are one-dimensional studies, looking at either the time or place of occurrence, and some of them have studied the prediction of occurrence as two-dimensional (Papazachos 1992; Rikitake 1976; Kelleher et al. 1973; Nanjo et al. 2006; Qin et al. 1999). While forecasting time or place alone cannot help prevent devastation caused by earthquakes, it is helpful if both dimensions are looked at together; it is useful to consider the dimension of magnitude as the third dimension along them. Some researchers have explained that the occurrence of earthquakes can be considered as memoryless models such as Markov models (Altinok and Kolcak 1999; Patwardhan et al. 1980; Nava et al. 2005). Some researchers have studied on earthquake prediction using a Markov chain model (such as Di Luccio et al. 1997; Console et al. 2002; Console 2001), and few of them have considered the model as semi-Markov (Altinok and Kolcak 1999; Patwardhan et al. 1980). These papers using a semi-

Correspondence: ramin_sadeghian@yahoo.com

Islamic Azad University, South Tehran Branch, Industrial Engineering Faculty, Tehran, Iran
Markov model don't have either much analysis or any evaluation on obtained results. Altinok and Kolcak (1999) and Patwardhan et al. (1980) didn't use scientific techniques for selecting used regions and magnitudes, while we apply a clustering method for regions and magnitudes. Altinok and Kolcak (1999) didn't discuss about selecting time unit. Nava et al. (2005) used a Markov model for the validation of earthquake occurrence, while the proposed model is a semi-Markov model. Patwardhan et al. (1980) and Altinok and Kolcak (1999) didn't forecast time, place, and magnitude of earthquake occurrence simultaneously, while we obtain joint functions for forecasting all dimensions simultaneously. The paper here tries to improve weaknesses of the papers and apply different methods for determining regions, magnitudes, and time unit. Using a semi-Markov model to forecast time, place, and magnitude of occurrence of earthquakes in the province, it is possible to improve the precautionary measures. Some advantages of semi-Markov models in comparison to other models were explained by Patwardhan et al. (1980). Because energies saved under faults are freed when an earthquake occurs, the time and magnitude of the earthquake within a specified region depend on the previous earthquake in the region. Consequently, earthquake occurrence can be explained by Markov models. Note that future periods can be forecasted by previous periods in Markov models. Nava et al. (2005) used a Markov model for the validation of earthquake, 
while the proposed model is a semi-Markov model. Also, Sadeghian and Jalali-Naini (2008a,b) applied a semi-Markov model and probability distributions on the field of earthquakes. In the next sections, we describe definitions of a semi-Markov model and its necessary parameters such as holding time, core matrix, interval transition probability matrix, and their analyses. A semi-Markov model is used for Tehran province. Next, the data corresponding to the province and its magnitudes during 1966 to 2004 are clustered, and then, both region-to-region and magnitude-to-magnitude transition matrices are obtained. Forecasting is performed by matrices obtained during next 3 years.

\section{Case description}

\section{Semi-Markov model}

Consider a continuous time chain $\{X(t)\}_{t \in[0, \infty)}$, taking value in a discrete state space $S$ :

1. $T_{n}(n \geq 1)$ is the time of the $n$th transition after $t=0$. (Note that the chain does not have to change states at each transition, as at time $T_{4}$ in Figure 1 ).

2. The duration between two consecutive transitional epochs $T_{n-1}, T_{n}$ is defined as the chain's $n$th holding time.

3. If the chain's sample path is right continuous (i.e., the time is considerable as piecemeal), then we write $X_{n} \equiv X\left(T_{n}\right)$.

The chain of $\{X(t)\}_{t \in[0, \infty)}$ is said to be semi-Markov if its development after each transition is independent of its behavior before that time. This means that the distribution of its holding time $T_{n}-T_{n-1}$ is independent of its behavior before $T_{n-1}$ but may be a function of $X_{n-1}=X\left(T_{n-1}\right), X_{n}=X\left(T_{n}\right)$. If $X_{n-1}=i, X_{n}=j$, we denote the holding time $T_{n}-T_{n-1}$ by $t_{i j}$.

If all holding times in a semi-Markov chain are equal to a constant value, the chain can be studied as a discrete-time Markov chain. To describe it completely, we need only to have all transition probabilities.

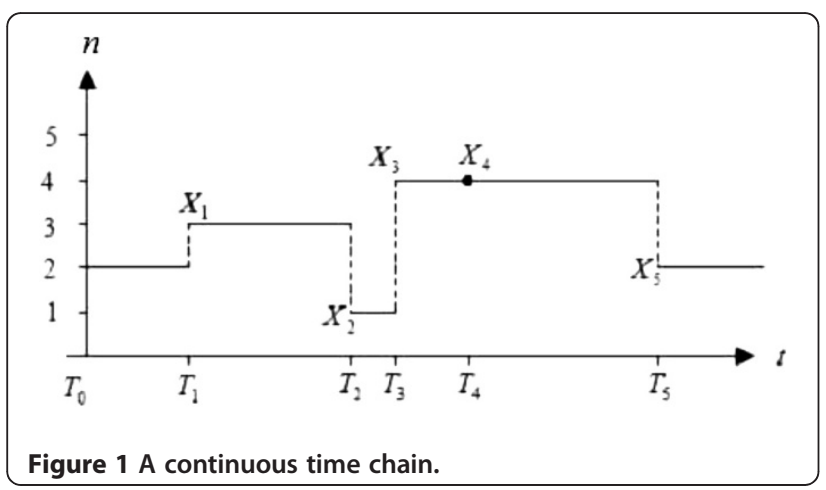

If a semi-Markov chain has only one state, all its holding times can only be a function of this one state; hence, they are independent and identically distributed. The chain therefore can be studied as a renewal stream, with a renewal at each transition; we only need its holding time distribution as the life distribution of the stream (Jalali-Naini 1997; Minh 2001). See previous studies of Kulkarni (1995), Cotea and Stein (2006), Bradley et al. (2006), Bradley and Wilson (2005), and Jenamani et al. (2003) for some general references about semi-Markov models.

\section{Transition matrix}

The transition probability $G_{i j}$ is the probability that a semi-Markov process which has entered state $i$ on its last transition will enter state $j$ on its next transition. These probabilities must satisfy the conditions:

$$
\begin{aligned}
& G_{i j} \geq 0 ; \quad i=1, \ldots, N, j=1, \ldots, N \\
& \sum_{j=1}^{N} G_{i j}=1 ; i=1, \ldots, N
\end{aligned}
$$

where $N$ is the total number of states in the system (Minh 2001; Altinok and Kolcak. 1999; Kulkarni 1995; Jenamani et al. 2003).

\section{Holding time}

Before the transition from state $i$ to state $j$, the process remains in state $i$ for a time $t_{i j}$. The holding times $t_{i j}$ are positive, integer-valued, random variables. All holding times are finite, and each is at least equal to one time unit. The probability mass function $T_{i j}$ in $t_{i j}$ is called the holding time mass function for a transition from state $i$ to $j$ :

$$
\operatorname{Pr}\left\{t_{i j}=m\right\}=T_{i j}(m) ; \quad m=1, \ldots, n
$$

Here, $n$ is the number of time intervals. We must specify both the holding time mass functions and the transition probabilities to describe a discrete-time semi-Markov process completely (see Minh 2001; Altinok and Kolcak 1999; Kulkarni 1995; Jenamani et al. 2003).

\section{Core matrix}

The $i$ th element of the core matrix $C(m)$ is the probability of the joint event in which a system that entered state $i$ at time 0 makes its next transition to state $j$ and makes that transition after a holding time $m$ (Altinok and Kolcak 1999).

$$
\begin{aligned}
& C_{i j}(m)=\operatorname{Pr}\left(\left(X_{n}=j \mid X_{n-1}=i\right),\left(t_{i j}=m\right)\right) \\
& \quad=\operatorname{Pr}\left(X_{n}=j \mid X_{n-1}=i\right) \cdot \operatorname{Pr}\left(t_{i j}=m\right) \\
& \quad=G_{i j} \cdot T_{i j}(m) \Rightarrow C_{i j}(m)=G_{i j} T_{i j}(m) ; i, j \\
& \quad=1, \ldots, N, m=1, \ldots, n
\end{aligned}
$$


We denote the above equation in congruent matrix multiplication form by,

$$
C(m)=G \otimes T(m),
$$

where the operator $\otimes$ denotes multiplication of corresponding elements. If we sum the elements of $C(m)$ across the $i$ throw, we obtain the waiting time mass function $w_{i}(m)$ for the $i$ th state:

$$
\sum_{j=1}^{N} C_{i j}(m)=\sum_{j=1}^{N} G_{i j} T_{i j}(m)=w_{i}(m),
$$

Indeed, $w_{i}(m)$ is the probability that waiting time for the $i$ th state is equal to $m$.

The cumulative probability distribution of the waiting time is obtained from

$$
\operatorname{LEw}_{i}(n)=\sum_{m=1}^{n} w_{i}(m)
$$

$L E w_{i}(m)$ is the probability that the waiting time for the $i$ th state is less than or equal to $n$. The complementary of $L E w_{i}$ is then given by

$$
G w_{i}(n)=\sum_{m=n+1}^{\infty} w_{i}(m)
$$

$G w_{i}(m)$ is the probability that waiting time for the $i$ th state is greater than $n$.

\section{Interval transition probability matrix}

The most important statistics of the semi-Markov process are the interval transition probabilities. The probability of a transition from state $i$ to state $j$ in the interval $(0, n)$ requires that the process makes at least one transition during that interval. The process could have made its first transition from state $i$ to some state at time $m$, where $(0 \leq m \leq n)$, and then by the sum of a succession of transitions, it could have made its way to state $j$ at time $n$.

$$
\begin{aligned}
F(n) & =G W(n)+\sum_{m=0}^{n} G \otimes T(m) F(n-m) \\
& =G W(n)+\sum_{m=0}^{n} C(m) F(n-m) ; \quad n \\
& =0,1,2, \ldots
\end{aligned}
$$

Here, $G W(n)$ is a diagonal matrix with its $i$ th element equal to $G w_{i}(n)$. The interval transition probability $F(n)$ is obtained by a recursive procedure. Because $T(0)$ is equal to $0, F(n)$ is obtained for the interval $1 \leq m \leq n$. In the case $n=0, F(n)$ is equal to the Kronecker Delta (Equation 9) or identity matrix.

$$
F_{i j}(0)= \begin{cases}1 & i=j \\ 0 & i \neq j\end{cases}
$$

In the earthquake phenomenon, these probabilities can be used for studying earthquake hazards (Altinok and Kolcak 1999; Patwardhan et al. 1980) and evaluating seismic hazard (Nava et al. 2005).

\section{Discussion and evaluation \\ Application of the model}

Tehran province is selected as an area of investigation. This area is bounded by longitudes $50.42^{\circ} \mathrm{E}, 53.16^{\circ} \mathrm{E}$ and latitudes $35.23^{\circ} \mathrm{N}, 36.33^{\circ} \mathrm{N}$ (Figure 2). Earthquake data are obtained from a catalog in the USGS website (USGS 2011).

After filtering and removing unsuitable data, the data wherein 44 earthquakes occurred from 1966 to 2004 are used. Note that if there are sufficient data such as a large number, accuracy, and long time interval, we expect that the accuracy of forecasting is increased and that the proposed model is validated mathematically.

We consider two transitions: from region-to-region and magnitude-to-magnitude.

\section{Region-to-region transition}

In the first stage, regions are chosen as states. We divided Tehran province into six regions $R_{1}, R_{2}, R_{3}, R_{4}, R_{5}, R_{6}$ (Figure 2). The transition probability matrix of region-toregion transitions was determined in Equation 10. Selecting 1 year for time unit, the largest time interval for the regionto-region transitions is found to be 5 years; therefore, holding time mass functions $T_{R}(m)$ are obtained by taking into account the time lapse between successive earthquakes (Equation 11). Also, interval transition probabilities $F_{R}(n)$ are calculated using Equation 8. They are shown in Equations 11 and 12 .

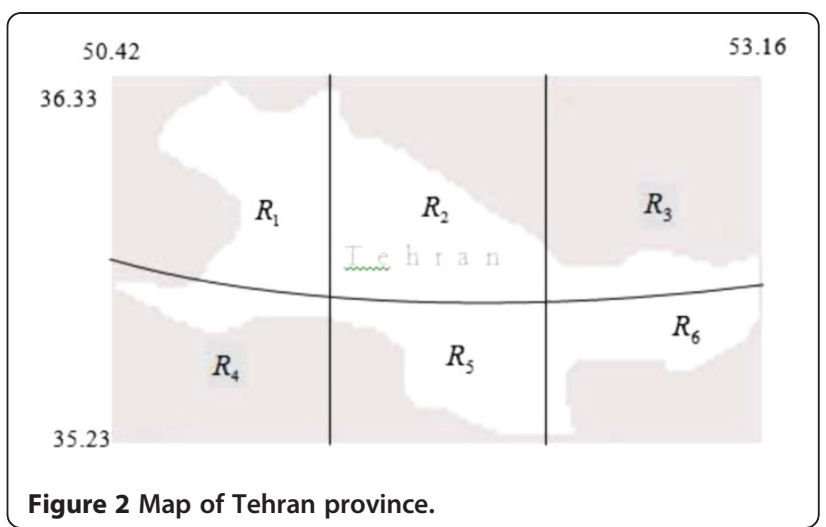




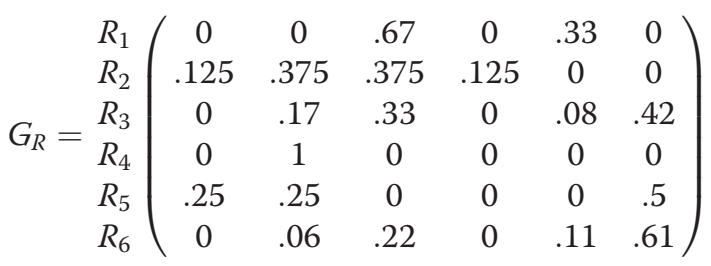

$$
T_{R}(1)=\left(\begin{array}{cccccc}
0 & 0 & .5 & 0 & 1 & 0 \\
1 & 1 & 1 & 1 & 0 & 0 \\
0 & 1 & 1 & 0 & 0 & .8 \\
0 & 1 & 0 & 0 & 0 & 0 \\
0 & 1 & 0 & 0 & 0 & .5 \\
0 & 1 & .5 & 0 & .5 & .91
\end{array}\right)
$$$$
T_{R}(2)=\left(\begin{array}{cccccc}
0 & 0 & 0 & 0 & 0 & 0 \\
0 & 0 & 0 & 0 & 0 & 0 \\
0 & 0 & 0 & 0 & 0 & .2 \\
0 & 0 & 0 & 0 & 0 & 0 \\
0 & 0 & 0 & 0 & 0 & .5 \\
0 & 0 & .25 & 0 & .5 & .09
\end{array}\right)
$$$$
T_{R}(3)=\left(\begin{array}{cccccc}
0 & 0 & .5 & 0 & 0 & 0 \\
0 & 0 & 0 & 0 & 0 & 0 \\
0 & 0 & 0 & 0 & 0 & 0 \\
0 & 0 & 0 & 0 & 0 & 0 \\
1 & 0 & 0 & 0 & 0 & 0 \\
0 & 0 & .25 & 0 & 0 & 0
\end{array}\right)
$$

$$
T_{R}(4)=\left(\begin{array}{llllll}
0 & 0 & 0 & 0 & 0 & 0 \\
0 & 0 & 0 & 0 & 0 & 0 \\
0 & 0 & 0 & 0 & 0 & 0 \\
0 & 0 & 0 & 0 & 0 & 0 \\
0 & 0 & 0 & 0 & 0 & 0 \\
0 & 0 & 0 & 0 & 0 & 0
\end{array}\right)
$$

$$
T_{R}(5)=\left(\begin{array}{cccccc}
0 & 0 & 0 & 0 & 0 & 0 \\
0 & 0 & 0 & 0 & 0 & 0 \\
0 & 0 & 0 & 0 & 1 & 0 \\
0 & 0 & 0 & 0 & 0 & 0 \\
0 & 0 & 0 & 0 & 0 & 0 \\
0 & 0 & 0 & 0 & 0 & 0
\end{array}\right)
$$

$F_{R}(1)=\left(\begin{array}{cccccc}.335 & 0 & .335 & 0 & .33 & 0 \\ .125 & .375 & .375 & .125 & 0 & 0 \\ 0 & .17 & .494 & 0 & 0 & .336 \\ 0 & 1 & 0 & 0 & 0 & 0 \\ 0 & .25 & 0 & 0 & .5 & .25 \\ 0 & .06 & .11 & 0 & .055 & .775\end{array}\right)$

$F_{R}(2)=\left(\begin{array}{cccccc}.335 & .14 & .165 & 0 & .165 & .195 \\ .089 & .329 & .368 & .047 & .041 & .126 \\ .021 & .14 & .345 & .021 & .018 & .455 \\ .125 & .375 & .375 & .125 & 0 & 0 \\ .031 & .109 & .121 & .031 & .264 & .444 \\ .008 & .088 & .193 & .008 & .113 & .59\end{array}\right)$

$F_{R}(3)=\left(\begin{array}{cccccc}.017 & .083 & .49 & .017 & .093 & .3 \\ .1 & .24 & .334 & .041 & .043 & .242 \\ .025 & .137 & .33 & .018 & .056 & .434 \\ .089 & .329 & .368 & .047 & .041 & .126 \\ .274 & .119 & .168 & .014 & .052 & .373 \\ .014 & .117 & .262 & .011 & .112 & .484\end{array}\right)$

$F_{R}(4)=\left(\begin{array}{cccccc}.1 & .142 & .331 & .01 & .036 & .381 \\ .06 & .193 & .356 & .03 & .054 & .307 \\ .03 & .133 & .35 & .017 & .073 & .397 \\ .1 & .24 & .334 & .041 & .043 & .242 \\ .114 & .11 & .281 & .015 & .15 & .33 \\ .035 & .129 & .274 & .015 & .096 & .451\end{array}\right)$

$F_{R}(5)=\left(\begin{array}{cccccc}.055 & .128 & .325 & .018 & .08 & .394 \\ .058 & .17 & .348 & .025 & .054 & .342 \\ .033 & .13 & .29 & .017 & .155 & .375 \\ .06 & .193 & .356 & .03 & .054 & .307 \\ .111 & .144 & .264 & .014 & .107 & .36 \\ .051 & .132 & .288 & .016 & .086 & .427\end{array}\right)$

\section{Magnitude-to-magnitude transitions}

In the second stage, magnitudes are chosen as states. Similar to the previous stage, we apply the AGNES method (Ghazanfari and Rezaei 2006) for the clustering of magnitudes, and three clusters on magnitudes as states are established in the whole regions as follows:

$$
\begin{aligned}
& M 1: \mathrm{MB}<4.5 \\
& M 2: 4.5 \leq \mathrm{MB} \leq 5 \\
& M 3: 5<\mathrm{MB}
\end{aligned}
$$

where MB is a unit of the magnitude of earthquakes; it is called as body wave magnitude.

The transition matrix of magnitude-to-magnitude transitions is given in Equation 17. The holding time mass functions $T_{M}(m)$ and interval transition probabilities 
$F_{M}(n)$ are calculated using Equation 2 through Equation 8. These are shown in Equations 15 and 16.

$$
\begin{aligned}
G_{M}= & \begin{array}{l}
M_{1} \\
M_{2} \\
M_{3}
\end{array}\left(\begin{array}{ccc}
.5 & .3 & .2 \\
.45 & .5 & .05 \\
.167 & .5 & .333
\end{array}\right) \\
T_{M}(1) & =\left(\begin{array}{ccc}
1 & 1 & .75 \\
.67 & .7 & 0 \\
0 & 1 & 1
\end{array}\right) T_{M}(2)=\left(\begin{array}{ccc}
0 & 0 & .25 \\
.11 & .2 & 0 \\
1 & 0 & 0
\end{array}\right) \\
T_{M}(3) & =\left(\begin{array}{ccc}
0 & 0 & 0 \\
.22 & 0 & 1 \\
0 & 0 & 0
\end{array}\right) \quad T_{M}(4)=\left(\begin{array}{lll}
0 & 0 & 0 \\
0 & 0 & 0 \\
0 & 0 & 0
\end{array}\right) \\
T_{M}(5) & =\left(\begin{array}{lll}
0 & 0 & 0 \\
0 & .1 & 0 \\
0 & 0 & 0
\end{array}\right)
\end{aligned}
$$

$$
\begin{aligned}
F_{M}(1) & =\left(\begin{array}{ccc}
.55 & .3 & .15 \\
.302 & .698 & 0 \\
0 & .5 & .5
\end{array}\right) F_{M}(2)=\left(\begin{array}{lll}
.365 & .435 & .2 \\
.322 & .633 & .045 \\
.318 & .515 & .167
\end{array}\right) \\
F_{M}(3) & =\left(\begin{array}{lll}
.326 & .51 & .164 \\
.38 & .486 & .134 \\
.359 & .538 & .103
\end{array}\right) F_{M}(4)=\left(\begin{array}{ccc}
.347 & .507 & .146 \\
.336 & .514 & .15 \\
.37 & .495 & .135
\end{array}\right) \\
& \\
F_{M}(5) & =\left(\begin{array}{lll}
.347 & .51 & .143 \\
.328 & .526 & .146 \\
.346 & .507 & .147
\end{array}\right)
\end{aligned}
$$

\section{Forecasting}

In this section, time and magnitude of earthquake occurrence are forecasted for each region in the next 3 years. Note that some forecasting methods based on probability approaches don't have sufficient accuracy, and others are more accurate. Matrices $P_{1}$ through $P_{6}$ show region-to-region transition probabilities by time of occurrence without considering the dimension of magnitude. In other words, the sum of each column is equal to 1 (see Tables 1,2 , $3,4,5$, and 6). For example, the $i j$ th element of matrix $P_{1}$ shows the probability of earthquake occurrence in region $R_{i}$ during year $j$ if the last earthquake has occurred in region $R_{1}$. Similarly, matrices $P_{7}$ through $P_{9}$ show magnitude-tomagnitude transition probabilities by time of occurrence without considering the spatial dimension (see Tables 7, 8, 9). In these matrices, the sum of each column is also equal to 1 .

\begin{tabular}{|c|c|c|c|c|c|c|}
\hline & & 1 & 2 & 3 & 4 & 5 \\
\hline \multirow[t]{6}{*}{$P 2=$} & $R 2$ to $R 1$ & 0.125 & 0.089 & 0.1 & 0.06 & 0.058 \\
\hline & $R 2$ to $R 2$ & 0.0375 & 0.329 & 0.24 & 0.193 & 0.17 \\
\hline & $R 2$ to $R 3$ & 0.375 & 0.368 & 0.334 & 0.356 & 0.348 \\
\hline & $R 2$ to $R 4$ & 0.125 & 0.047 & 0.041 & 0.03 & 0.025 \\
\hline & $R 2$ to $R 5$ & 0 & 0.041 & 0.043 & 0.054 & 0.054 \\
\hline & $R 2$ to $R 6$ & 0 & 0.126 & 0.242 & 0.307 & 0.342 \\
\hline
\end{tabular}

Here, a semi-Markov model for considering the time of earthquake occurrence is applied. This method requires time, place, and magnitude of previous
Table 1 Matrix $P 1$

\begin{tabular}{lcccccc}
\hline & $\mathbf{1}$ & $\mathbf{2}$ & $\mathbf{3}$ & $\mathbf{4}$ & $\mathbf{5}$ \\
\hline$P 1=$ & $R 1$ to $R 1$ & 0.335 & 0.335 & 0.017 & 0.1 & 0.055 \\
$R 1$ to $R 2$ & 0 & 0.14 & 0.083 & 0.142 & 0.128 \\
$R 1$ to $R 3$ & 0.335 & 0.165 & 0.49 & 0.331 & 0.325 \\
$R 1$ to $R 4$ & 0 & 0 & 0.017 & 0.01 & 0.018 \\
$R 1$ to $R 5$ & 0.33 & 0.165 & 0.093 & 0.036 & 0.08 \\
$R 1$ to $R 6$ & 0 & 0.195 & 0.3 & 0.381 & 0.394 \\
\hline
\end{tabular}

Table 2 Matrix $\mathbf{P 2}$

Table 3 Matrix P3

\begin{tabular}{rcccccc}
\hline$P 3=$ & $\mathbf{1}$ & $\mathbf{2}$ & $\mathbf{3}$ & $\mathbf{4}$ & $\mathbf{5}$ \\
\hline$R 3$ to $R 1$ & 0 & 0.021 & 0.025 & 0.03 & 0.033 \\
$R 3$ to $R 2$ & 0.17 & 0.14 & 0.137 & 0.133 & 0.13 \\
$R 3$ to $R 3$ & 0.494 & 0.345 & 0.33 & 0.35 & 0.29 \\
$R 3$ to $R 4$ & 0 & 0.021 & 0.018 & 0.017 & 0.017 \\
$R 3$ to $R 5$ & 0 & 0.018 & 0.056 & 0.073 & 0.155 \\
$R 3$ to $R 6$ & 0.336 & 0.455 & 0.434 & 0.397 & 0.375 \\
\hline
\end{tabular}

Table 4 Matrix P4

\begin{tabular}{llccccc}
\hline & $\mathbf{1}$ & $\mathbf{2}$ & $\mathbf{3}$ & $\mathbf{4}$ & $\mathbf{5}$ \\
\hline$P 4=$ & $R 4$ to $R 1$ & 0 & 0.125 & 0.089 & 0.1 & 0.06 \\
$R 4$ to $R 2$ & 1 & 0.375 & 0.329 & 0.24 & 0.193 \\
$R 4$ to $R 3$ & 0 & 0.375 & 0.368 & 0.334 & 0.356 \\
$R 4$ to $R 4$ & 0 & 0.125 & 0.047 & 0.041 & 0.03 \\
$R 4$ to $R 5$ & 0 & 0 & 0.041 & 0.043 & 0.054 \\
$R 4$ to $R 6$ & 0 & 0 & 0.126 & 0.242 & 0.307 \\
\hline
\end{tabular}

Table 5 Matrix P5

\begin{tabular}{lcccccc}
\hline & $\mathbf{1}$ & $\mathbf{2}$ & $\mathbf{3}$ & $\mathbf{4}$ & $\mathbf{5}$ \\
\hline$P 5=$ & $R 5$ to $R 1$ & 0 & 0.031 & 0.274 & 0.114 & 0.111 \\
$R 5$ to $R 2$ & 0.25 & 0.109 & 0.119 & 0.11 & 0.144 \\
$R 5$ to $R 3$ & 0 & 0.121 & 0.168 & 0.281 & 0.264 \\
$R 5$ to $R 4$ & 0 & 0.031 & 0.014 & 0.015 & 0.014 \\
$R 5$ to $R 5$ & 0.5 & 0.264 & 0.052 & 0.15 & 0.107 \\
$R 5$ to $R 6$ & 0.25 & 0.444 & 0.373 & 0.33 & 0.36 \\
\hline
\end{tabular}


Table 6 Matrix P6

\begin{tabular}{|c|c|c|c|c|c|c|}
\hline & & 1 & 2 & 3 & 4 & 5 \\
\hline \multirow[t]{6}{*}{$P 6=$} & $R 6$ to $R 1$ & 0 & 0.008 & 0.014 & 0.035 & 0.051 \\
\hline & $R 6$ to $R 2$ & 0.06 & 0.088 & 0.117 & 0.129 & 0.132 \\
\hline & $R 6$ to $R 3$ & 0.11 & 0.193 & 0.262 & 0.274 & 0.288 \\
\hline & $R 6$ to $R 4$ & 0 & 0.008 & 0.011 & 0.015 & 0.016 \\
\hline & $R 6$ to $R 5$ & 0.055 & 0.113 & 0.112 & 0.096 & 0.086 \\
\hline & $R 6$ to $R 6$ & 0.775 & 0.59 & 0.484 & 0.451 & 0.427 \\
\hline
\end{tabular}

Table 7 Matrix P7

\begin{tabular}{|c|c|c|c|c|c|c|}
\hline & & 1 & 2 & 3 & 4 & 5 \\
\hline \multirow[t]{3}{*}{$P 7=$} & $M 1$ to $M 1$ & 0.55 & 0.365 & 0.326 & 0.347 & 0.347 \\
\hline & $M 1$ to $M 2$ & 0.3 & 0.435 & 0.51 & 0.507 & 0.51 \\
\hline & $M 1$ to $M 3$ & 0.15 & 0.2 & 0.164 & 0.146 & 0.143 \\
\hline
\end{tabular}

Table 8 Matrix $\mathbf{P 8}$

\begin{tabular}{|c|c|c|c|c|c|c|}
\hline & & 1 & 2 & 3 & 4 & 5 \\
\hline \multirow[t]{3}{*}{$P 8=$} & $M 2$ to $M 1$ & 0.302 & 0.322 & 0.38 & 0.336 & 0.328 \\
\hline & $M 2$ to $M 2$ & 0.698 & 0.633 & 0.486 & 0.514 & 0.526 \\
\hline & $M 2$ to $M 3$ & 0 & 0.045 & 0.134 & 0.15 & 0.146 \\
\hline
\end{tabular}

Table 9 Matrix P9

\begin{tabular}{|c|c|c|c|c|c|c|}
\hline & & 1 & 2 & 3 & 4 & 5 \\
\hline \multirow[t]{3}{*}{$P 9=$} & $M 3$ to $M 1$ & 0 & 0.318 & 0.359 & 0.37 & 0.346 \\
\hline & $M 3$ to $M 2$ & 0.5 & 0.515 & 0.538 & 0.495 & 0.507 \\
\hline & $M 3$ to $M 3$ & 0.5 & 0.167 & 0.103 & 0.135 & 0.147 \\
\hline
\end{tabular}

earthquakes. If the previous earthquake is within region $r_{0}$ and its magnitude is $m_{0}$, then the probability of occurrence of the next earthquake within region $r_{1}$ and magnitude $m_{1}$ after $d$ time periods is a conditional probability defined as follows:

$$
\begin{aligned}
& P\left(R=r_{1}, M=m_{1} \mid r_{0}, m_{0}, d\right)=P\left(r_{1}, m_{1} \mid r_{0}, m_{0}, d\right) \\
& =\stackrel{\text { region }}{=}=\underset{\text { magnitude }- \text { to }- \text { magnitude }}{=}=\stackrel{=}{=}=\stackrel{\text { region }}{=}=\stackrel{=}{=}=\stackrel{\text { transition }}{=}=\underset{\text { probabilities }}{=}=\underline{=}=\stackrel{=}{=}= \\
& \mathrm{P}\left(r_{1} \mid r_{0}, d\right) \cdot P\left(m_{1} \mid m_{0}, d\right)=F_{R_{r_{0} r_{1}}}(d) . F_{M_{m_{0} m_{1}}}(d)
\end{aligned}
$$

Using the above equations and the list of data, the last earthquake in Tehran province occurred at 28 May 2004 within region $R_{1}$ with a magnitude of 6.3 (MB). Consider the current time as November 2006; in this way, the proposed model forecasts that the most probable occurrence of an earthquake within
Tehran province within region $R_{3}$ (northeast of Tehran province) with a magnitude of $M_{2}(4.5 \leq M B \leq 5)$ from May 2006 to May 2007 is 0.2636 (26.36\%).

\section{Conclusion}

This article attempts to forecast the likelihood of all earthquakes within Tehran province in the next years by a semi-Markov model. The proposed model forecasts that the most probable occurrence of an earthquake within Tehran province within region $R_{3}$ (northeast of Tehran province) with a magnitude of $M_{2} \quad(4.5 \leq \mathrm{MB} \leq 5)$ from May 2006 to May 2007 is 0.2636 (26.36\%). According to our forecasting, an earthquake occurred in Roudehen (northeast of Tehran province) with a magnitude of $4.2 \mathrm{MB}$ on 20 December 2006. This can be considered as the evaluation of the proposed model.

The average magnitude of earthquake occurrence in Tehran province is $3.1 \mathrm{MB}$ yearly. The most number of earthquake occurrences have occurred in intervals $(4<\mathrm{MB} \leq 4.5)$. It includes $32 \%$ all of the events. Here, in this paper, the spatial, temporal, and magnitude dimensions are considered, while in past researches, these three dimensions were considered less.

As a conclusion, the amount of forecast errors shows that MAPE (Haj Shirmohammadi 2003) in this model is equal to $4.45 \%$, while in other models, it is more than 10\% (Papazachos 1992; Karakaisis 1994).

The obtained results during the next 50 days show that in other models, $42 \%$ of earthquakes have been correctly forecasted. The places of $16 \%$ of earthquake occurrences have been correctly forecasted, but not their magnitudes. Sixteen percent of the forecasted earthquakes occurred in places adjacent to the forecasted ones, but their magnitudes were true, and $26 \%$ of earthquakes could not have been forecasted at all.

In this model, $56 \%$ of earthquakes have been correctly forecasted in all the three dimensions. The places of $19 \%$ of earthquake occurrences have been correctly forecasted, but not their magnitudes. Nineteen percent of the forecasted earthquakes occurred in places adjacent to the forecasted ones, but their magnitudes were true, and $6 \%$ of earthquakes could not have been forecasted at all.

The mentioned statistics above show that this model in comparison to others can be applied in a more accurate forecasting.

Received: 10 March 2010 Accepted: 2 March 2012

Published: 31 August 2012

\section{References}

Altinok Y, Kolcak D (1999) An application of the semi-Markov model for earthquake occurrences in North Anatolia, Turkey. Journal of Balkan Geophysical Society 2:90-99 
Bradley JT, Wilson HJ (2005) Iterative convergence of passage-time densities in semi-Markov performance models. Performance Evaluation 60:237-254

Bradley JT, Dingle NJ, Harrison PG, Knottenbelt WJ (2006) Distributed computation of transient state distributions and passage time quantiles in large semi-Markov models. Future Generation Computer Systems 22:828-837

Console R (2001) Testing earthquake forecast hypotheses. Tectonophysics 338:261-268

Console R, Pantosti D, D'Addezio G (2002) Probabilistic approach to earthquake prediction. Ann Geophys 45(6):723-731

Cotea MJ, Stein WE (2006) A stochastic model for a visit to the doctor's office. Mathematical and Computer Modeling 45:309-323

Di Luccio F, Console R, Imoto M, Murru M (1997) Analysis of short time-space range seismicity pattern in Italy. Ann Geophys 40:4

Ghazanfari M, Rezaei M (2006) Introduction on fuzzy theory and its applications. IUST Press

Haj Shirmohammadi A (2003) Principle of production and inventory planning and control. Arkan Press, Isfahan

Jalali-Naini SG (1997) Stochastic processes and its applications in biology, agriculture, management and industries. Statistics and Information Office Press, Tehran

Jenamani M, Mohapatra PKJ, Ghose S (2003) A stochastic model of e-customer behavior. Electronic Commerce Research and Applications 2:81-94

Karakaisis GF (1994) Long-term earthquake prediction in Iran based on the time- and magnitude-predictable model. Physics of the Earth and Planetary Interiors 83:129-145

Kelleher J, Sykes L, Oliver J (1973) Possible criteria for predicting earthquake locations and their application to major plate boundaries of the Pacific and the Caribbean. Journal of Geophysical Research 78(14):2547-2585

Kulkarni VG (1995) Modeling and analysis of stochastic systems. Chapman \& Hall, London

Minh DL (2001) Applied probability models. Duxbury, Thomson Learning Press, California, pp 225-246

Nanjo KZ, Holliday JR, Chen C, Rundle JB, Turcotte DL (2006) Application of a modified pattern informatics method to forecasting the locations of future large earthquakes in the central Japan. Tectonophysics 424:351-366

Nava FA, Herrera C, Frez J, Glowacka E (2005) Seismic hazard evaluation using Markov chains: application to the Japan area. Pure and Applied Geophysics 162:1347-1366

Papazachos BC (1992) A time- and magnitude- predictable model for generation of shallow earthquakes in the Aegean area. Pure and Applied Geophysics 138(2):287-308

Patwardhan AS, Kulkarni RB, Tocher D (1980) A semi-Markov model for characterizing recurrence of great earthquakes. Bulletin of the Seismological Society of America 70(1):323-347

Qin C, Papadimitriou EE, Papazachos BC, Karakaisis GF (1999) On the validity of the regional time and magnitude predictable model in China. Ann Geophys 42(5)

Rikitake T (1976) Recurrence of great earthquakes at subduction zones. Tectonophysics 35:335-362

Sadeghian R (2007) What ever you don't know about earthquakes. Shooka Press, Tehran

Sadeghian R, Jalali-Naini GR (2008a) A new probability density function in earthquake occurrences. Journal of Industrial Engineering International 4(6):1-6

Sadeghian R, Jalali-Naini GR (2008b) Applying semi-Markov models for forecasting the triple dimensions of next earthquake occurrences. Dynamics of Continuous, Discrete and Impulsive Systems, Series B. Applications \& Algorithms 15(3b):395-416

USGS (2011) Earthquake hazards program http://neic.usgs.gov/neis/epic/epic.html

doi:10.1186/2251-712X-8-20

Cite this article as: Sadeghian: Forecasting time and place of earthquakes using a Semi-Markov model (with case study in

Tehran province). Journal of Industrial Engineering International 2012 8:20.

Submit your manuscript to a SpringerOpen ${ }^{\circ}$ journal and benefit from:

- Convenient online submission

- Rigorous peer review

- Immediate publication on acceptance

- Open access: articles freely available online

- High visibility within the field

- Retaining the copyright to your article

Submit your next manuscript at $\gg$ springeropen.com 